\title{
Modeling the defrost process in complex geometries - Part 1: Development of a one-dimensional defrost model
}

\author{
Simon van Buren ${ }^{1}$, Ellen Hertle ${ }^{1}$, Patric Figueiredo ${ }^{1}$, Reinhold Kneer $^{1}$, and Wilko Rohlfs ${ }^{1, *}$ \\ ${ }^{1}$ RWTH Aachen University, Institute of Heat and Mass Transfer, Augustinerbach 6, 52062 Aachen, \\ Germany
}

\begin{abstract}
Frost formation is a common, often undesired phenomenon in heat exchanges such as air coolers. Thus, air coolers have to be defrosted periodically, causing significant energy consumption. For the design and optimization, prediction of defrosting by a CFD tool is desired. This paper presents a one-dimensional transient model approach suitable to be used as a zero-dimensional wall-function in CFD for modeling the defrost process at the fin and tube interfaces. In accordance to previous work a multi stage defrost model is introduced (e.g. $[1,2])$. In the first instance the multi stage model is implemented and validated using MATLAB. The defrost process of a one-dimensional frost segment is investigated. Fixed boundary conditions are provided at the frost interfaces. The simulation results verify the plausibility of the designed model. The evaluation of the simulated defrost process shows the expected convergent behavior of the three-stage sequence.
\end{abstract}

\section{Introduction}

The phenomenon of frost formation occurs in various technical applications including the cooling of humid air such as air fin coolers. Frost is the deposition of water onto cold surfaces as porous structures of ice crystals [1]. The requirements for frost formation are that the substrate surface temperature is below the freezing temperature of water and that the temperature of the moist ambient air is below the dew point. According to Hayashi [3], the process of frost formation can be divided into three characteristic periods: crystal growth, frost layer growth, and full frost layer growth. Further, a preceding phase of nucleation is named as the initial period of frost formation. The characteristics of frost are closely related to the physical processes during the formation periods. The complex ramified structures of the frost layer lead to local inhomogeneities in properties (e.g. porosity, density, specific heat capacity) through the frost layer. The process of frost formation is experimentally and numerically widely studied (e.g. [4, 5]).

In technical applications, isolating frost layers reduce the cooling capacity of heat exchangers due to the low heat conductivity of frost. To maintain the heat transfer performance, the temperature gradient in the heat exchanger must be increased, reducing the energy efficiency of the device. Simultaneously, a frost layer reduces the air flow rate

\footnotetext{
*Corresponding author: rohlfs@wsa.rwth-aachen.de
} 
through the heat exchanger. At a certain point, the flow rate becomes too low to ensure stable operation conditions and only a strong increase in energy consumption allows for the continuation of the cooling mode. Consequently, the frost layer on cooled surfaces cannot grow indefinitely but must be removed regularly. Electric defrosting and defrosting by hot gaseous refrigerants are most commonly used in industrial applications [6, 7].

\section{Existing models for defrosting}

One of the first analytical approximations of non-stationary and stochastic [8] defrost processes was conducted by Sanders [1]. He discussed defrosting of a thin frost layer and formulated two different melting models based on a one-dimensional heat balance within the layer. In the first stage, the temperature of the frost layer increases due to wall-side heating. This process continues until the frost temperature at the wall-frost interface reaches the melting temperature. Further heating results in a phase change with a decreasing frost layer thickness. For this process, Sanders [1] presented two different modeling approaches (Fig. 1).

The first model approach assumes a constant temperature throughout the frost layer, a constant heat flux from the wall to the frost and a constant heat flux from the frost to the ambient air. Sanders [1] assumes a frost layer continuously attached to the wall. Consequently, the frost/air interface moves towards the wall. The second model approach accounts for a developing air gap $\delta_{\mathrm{g}}$ between the wall and the frost layer, causing additional thermal resistance and thus slowing down the defrost process. Furthermore, meltwater is assumed to diffuse into the remaining frost layer, where it refreezes at the opposite interface due to heat loss to the ambient. Note that the two model approaches presented by Sanders describe two limiting cases. The defrosting does not proceed homogeneously and the detachment of the frost layer will only occur locally and not on the entire substrate surface [9].
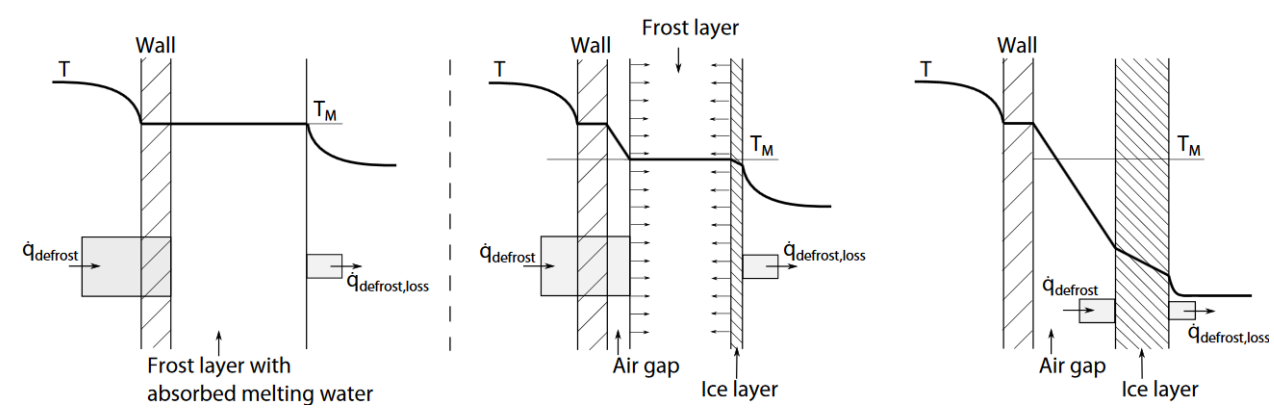

Fig. 1. Illustration of the defrost model by Sanders [1]. Alternative A: Absorption model (left), Alternative B: Air gap model (middle, right).

Machielsen [10] introduces a method to design an optimally balanced refrigeration system based on the model of Sanders [1]. They only consider the second alternative with an air gap between substrate surface and frost layer and divide the defrosting process into three phases.

Hoffenbecker [11] developed a transient numerical model to simulate the hot gas defrost cycle of an industrial air-cooling evaporator, using a simplified circular geometry. The model estimates the time and energy input for defrosting. 
Dopazo [2] propose a transient model for defrosting of a round-tube plate fin heat exchanger. The defrost process is divided into six different stages, as shown in Fig. 2. Different governing equations are formulated for a representative tube and fin element for each stage. The model is validated using experimental data.

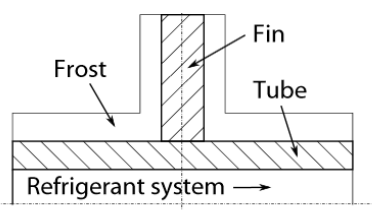

(a) Preheating

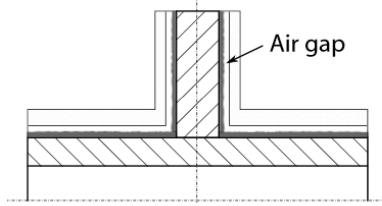

(d) Air gap appears

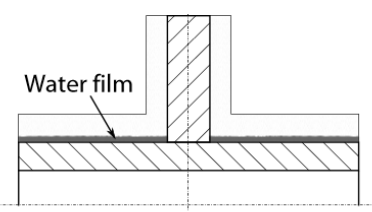

(b) Tube frost melting starts

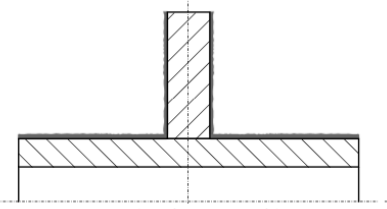

(e) Tube-fin water film

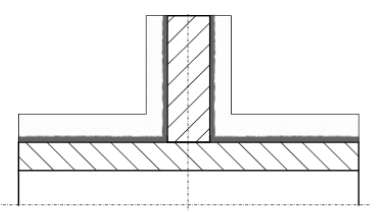

(c) Fin frost melting starts

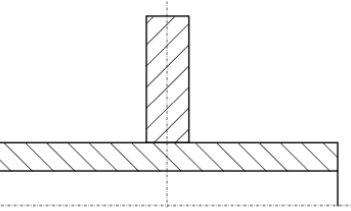

(f) Dry heating

Fig. 2. Six stages of defrosting according to Dopazo [2].

Wellig [12] developed a model to calculate the simultaneous heat and mass transfer during the melting of ice and frost deposits. To consider the changing air temperature and humidity along the fin, a stepwise calculation for several small elements along the fin depth was employed.

The defrost process on an electrically heated cylindrical tube has been analyzed by Sherif [13]. In their quasi-steady state approach, the frost melts at the heated surface and is immediately drained. The frost remains attached to the tube, while the frost surface moves towards the heated tube wall.

Alebrahim [6] presents a two-dimensional electric defrost model for a tube fitted with circular disk fins. The analysis is divided into two stages. First the frost formation stage is analyzed to calculate the temperature distribution within the tube and the frost. Secondly, the defrosting is and further divided into two sub-phases, a pre-melting phase and a melting phase. The author uses the enthalpy method to calculate the defrost time and the temperature distribution within the frost layer during the defrost process. In contrast to prior approaches the model allows to determine the temperature distribution in a two-dimensional space.

Experimental data on the defrost process and detailed research on various defrost methods has been conducted in Switzerland within the framework "Improvement of the Defrosting Process using Air-Cooled Evaporators" [14-16].

Mohs [9] developed a multi-stage defrost model which predicts the transient heat and mass transfer rates for a vertical, plain fin. The defrost process is divided into three separate stages, with each stage solved separately. The final state of each stage is used as an initial condition for the subsequent stage.

Nasr [17] experimentally studies the performance of air-to-air heat exchangers for ventilation purpose throughout the frosting and defrosting process. For the evaluation of the energy consumption, the crucial defrost time ratio is introduced. The influence of the fin-and-tube heat exchanger surface characteristics on the frosting and defrosting (e.g. required energy for the frost melting) is experimentally investigated by Wang [18]. 


\section{Design and model for defrosting}

We assume a frost layer of thickness $\delta$ normal to the substrate surface that is exposed to an ambient air flow, heating or cooling the frost layer from above. To reduce the computational effort simulating the complex defrosting process, some simplifications are necessary. Lateral heat conduction in the frost layer is assumed to be negligible compared to heat transport in normal direction (valid for thin frost layers or frost layers with homogenous boundary conditions). Secondly, mass transfer by diffusion within the frost layer and at the interface is the neglected. For implementation as a wall-function in a CFD solver (OpenFOAM) the frost layer is modeled with no physical expansion into the air region.

The above-mentioned simplifications allow for a wall-function formulation of the entire defrost process introducing an enhanced thermal coupling of the air and wall region. The thermal coupling condition of the wall and the frost region is the wall-frost interface temperature $T_{\mathrm{WF}}$ as well as the wall-side heat flux. In analogy, the temperature $T_{\mathrm{AF}}$ and the corresponding heat flux couple the frost and ambient air at the frost surface.

\subsection{Frost model}

The wall function model accounts for different scenarios of defrosting, including heating or cooling of the frost layer at the wall/frost boundary or the air/frost boundary. Like the models of Dopazo [2] and Hoffenbecker [7], the defrost process is subdivided into different characteristic stages. These stages include preheating, development of a water film, and the development of an air gap:

During the initial preheating phase the temperature in the entire frost layer is below the melting point $T_{\text {Melt }}$. Heat induction from the wall side causes melting and the development of a thin water film between the wall and the decreasing frost layer. When the water film exceeds a critical thickness, additional melt water is assumed to drain out, causing the development of an air gap between the water film (with the critical thickness) and the frost.

If the temperature of the frost layer reaches the melting point at the frost-air interface, air-side melting occurs. In this case, melt water is assumed to drain off, such that no additional resistance arises. A minimum frost layer thickness for the static stability is assumed. If this minimum thickness is surpassed, the remaining frost is assumed to drop off and the defrost process ends.

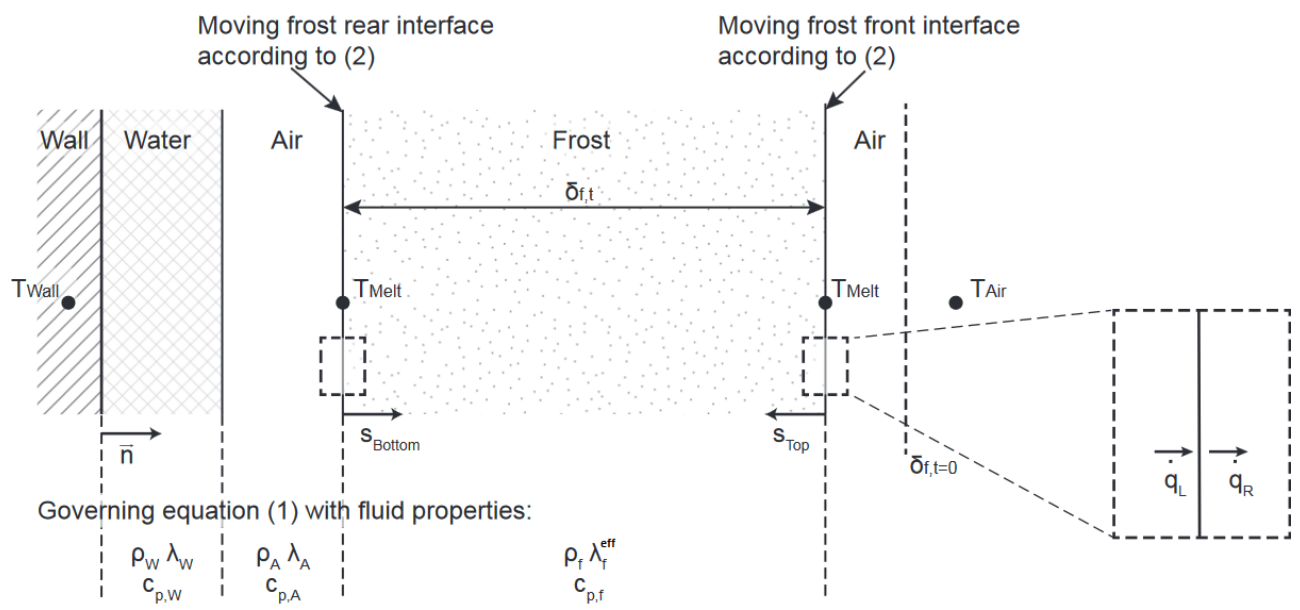

Fig. 3. Sketch of a frost layer with its governing equations and properties according to the region. 
A schematic of the different regions in the frost layer model is presented in Fig. 3. The temperature across the regions changes according to the one-dimensional energy equation

$$
\partial T_{\mathrm{f}} / \partial t=\lambda_{\mathrm{f}}{ }^{\text {eff }} /\left(c_{\mathrm{p}, \mathrm{f}} \rho_{\mathrm{f}}\right) \partial^{2} T_{\mathrm{f}} / \partial x^{2}
$$

Material properties, e.g. thermal conductivity $\lambda$, specific heat capacity $c_{\mathrm{p}}$, and density $\rho$ depend on the specific region (frost, water and air).

The frost layer is bounded by the two interfaces $S_{\text {Bottom }}$ and $S_{\text {Top. The two interface }}$ positions move in the melting phase according to

$$
\partial s_{\mathrm{f}} / \partial t=-\left(\left.q_{\mathrm{t}}\right|_{\mathrm{x}=\mathrm{sL}}+\left.q_{\mathrm{t}}\right|_{\mathrm{x}=\mathrm{sR}}\right) /\left(h_{\text {Melt }} \rho_{\mathrm{f}}\right)
$$

with $q_{\mathrm{t}}=-\lambda_{\mathrm{f}}^{\text {eff }} \partial T_{\mathrm{f}} / \partial x$. Consequently, a localized heat sink with a discontinuity in the heat flux $\left.q_{\mathrm{t}}\right|_{\mathrm{x}=\mathrm{sL}}$ and $\left.q_{\mathrm{t}}\right|_{\mathrm{x}=\mathrm{SR}}$ drives the phase change.

Required initial conditions are the frost layer thickness $\delta$, the local frost temperature $T(x)$ and the local frost porosity $\varepsilon(x)$ which is assumed to be constant over the defrost process (no densification). The porosity $\varepsilon$ is defined as the ratio of the frost pore volume $V_{\mathrm{p}}$ to the to the total volume $V_{\text {tot }}$. Initially, the frost interfaces $S_{\text {Bottom }}$ and $S_{\text {Top }}$ are located at the wall and the position $\delta$, respectively. For the boundary conditions, a time-dependent wall temperature $T_{\mathrm{WF}}(t)$ and ambient air temperature (at the frost-air interface), $T_{\mathrm{AA}}(t)$ are required. Due to the moving frost boundary edges described by eq. 2 , the system has two further degrees of freedom. Thus, the temperature at the melting interface is set to the melting point of the frost.

\subsection{Frost properties}

Frost layers are usually characterized by a strong inhomogeneity. Porous air and ice compositions change along the frost height leading to a local gradient in porosity. Frost properties are modeled in agreement to previous studies $[1,19,20]$. Density, $\rho_{\mathrm{f}}$, effective thermal conductivity, $\lambda_{\mathrm{f}}{ }^{\mathrm{eff}}$, and specific heat capacity, $c_{\mathrm{f}}$, of the frost layer are derived by the local frost porosity $\varepsilon$ and the physical properties of the components ice, air and water [20]:

$$
\begin{gathered}
\rho_{\mathrm{f}}=(1-\varepsilon) \rho_{\text {ice }}+\varepsilon\left(\rho_{\text {vapor }, \mathrm{f}}+\rho_{\text {air }, \mathrm{f}}\right) \approx(1-\varepsilon) \rho_{\text {ice }}+\varepsilon \rho_{\text {air. }} \\
\lambda_{\mathrm{f}}^{\text {eff }}=0.001202 \rho_{\mathrm{f}}^{0.963} \\
\rho_{\mathrm{f}} c_{\mathrm{f}}=\varepsilon \rho_{\mathrm{a}} c_{\mathrm{a}}+(1-\varepsilon) \rho_{\text {ice }} c_{\text {ice }} \approx(1-\varepsilon) \rho_{\text {ice }} c_{\text {ice }}
\end{gathered}
$$

\section{Numerical implementation}

The model has been implemented in MATLAB prior to an implementation as wall function in the CFD solver. The locations of the frost boundaries $S_{\text {Top }}$ and $S_{\text {Bottom }}$ are discretized in accordance to the underlying mesh of the frost section, avoiding complex mesh adjustments of moving grids. The individual cells are assigned the thermophysical properties of the containing material (water, frost, air) and changed accordingly throughout the course of the defrost process.

The computation of the presented set of differential equations is carried out by the ode $23 s$ solver provided by MATLAB. Discrete movement of the frost boundaries and the connected reallocation of cells is realized by event functions that are activated upon a complete shift of the frost interface to the neighboring mesh interface.

Investigating the mesh dependency, a converging behavior is observed, showing variations of $5 \%$ at 25 cells and $1 \%$ at 100 cells. Thus, a mesh resolution of 100 cells is used for the presented results. 


\section{Results}

The model approach outlined and described in the previous sections is first applied to study the defrost process of a one-dimensional homogeneous frost layer $(\varepsilon=0.5)$ of $1 \mathrm{~mm}$ of thickness with a linear temperature profile from 258 to $270 \mathrm{~K}$ a time invariant boundary conditions $\left(\mathrm{T}_{\text {Wall }}=281 \mathrm{~K}, \mathrm{~T}_{\text {Air }}=276 \mathrm{~K}, \alpha=25 \mathrm{~W} /\left(\mathrm{m}^{2} \mathrm{~K}\right)\right)$. The minimal frost- and maximal water-layer thickness are both $0.2 \mathrm{~mm}$.

\subsection{Dissection of the defrost process}

Figure 4 shows the temperature profiles of the melting frost layer at four instants of time. In the initial phase of the defrost process (at $t=0.1 \mathrm{~s}$, see Fig. $4 \mathrm{a}$ ) the temperature profile in the frost layer changes rapidly. Owing to the high temperature at the frost-wall interface, rapid melting occurs and a thin water layer develops within the first $0.1 \mathrm{~s}$. Consequently, the preheating phase ends with the beginning of melting and the development of a water film in less than $0.1 \mathrm{~s}$. The temperature minimum in the center of the frost layer indicates a strongly transient heat transfer process in the initial phase.

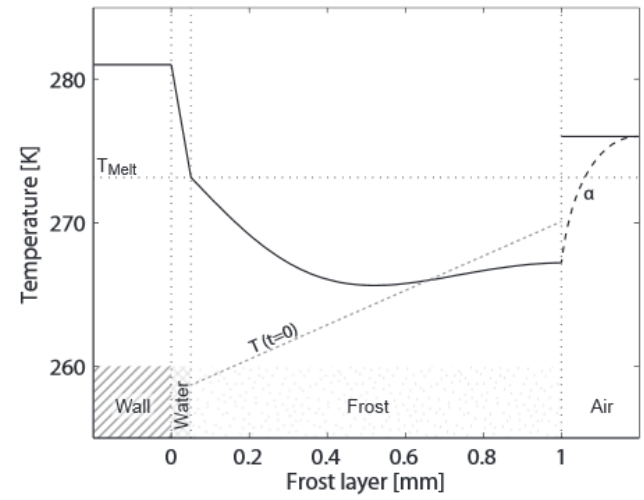

(a) Time $t=0.1 \mathrm{~s}$

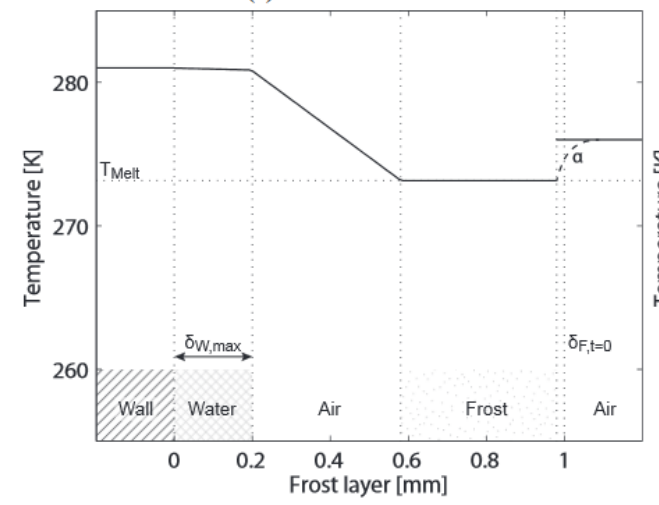

(c) Time $t=90 \mathrm{~s}$

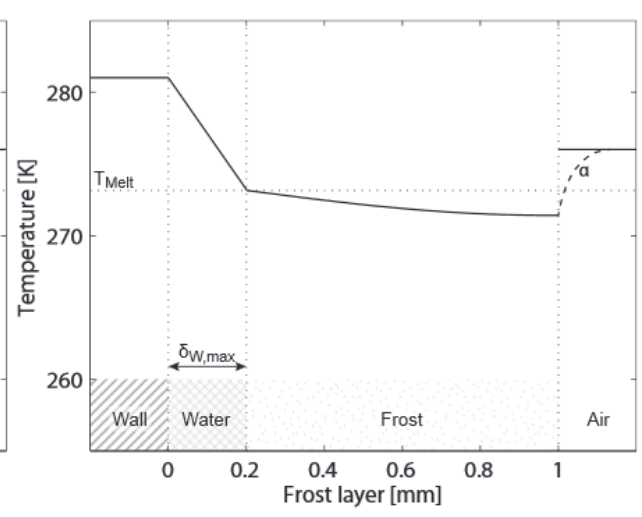

(b) Time $t=1.1 \mathrm{~s}$

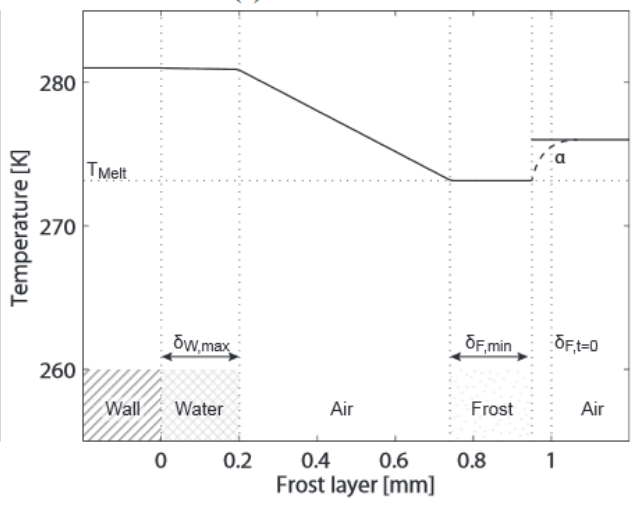

(d) Time $t=182.5 \mathrm{~s}$

Fig. 4. Temperature profiles of a defrost process at different times. Convection at the ambient air-frost interface is indicated by the dashed line labeled $\alpha$. Vertical dashed lines refer to the interfaces between the regions.

At $t=1.1 \mathrm{~s}$ (Fig. 4b), the water film has increased in size to $0.2 \mathrm{~mm}$ (critical water film thickness). The model assumes now that further melt water drains out, not remaining in the frost-wall gap - end of phase "development of a water film". The flattened temperature 
profile inside the frost layer and the straight profile in the water layer indicate a quasistationary defrost process.

At $t=90 \mathrm{~s}$ (Fig. 4c) an air gap has formed (phase "development of an air gap"). The size of the water film remains at the critical thickness of $0.2 \mathrm{~mm}$. Owing to the lower thermal conductivity of air compared to water and frost, the temperature drops across the air gap, indicating that the air gap presents the most significant resistance in the defrost process. In addition to the wall-sided melting process, air-sided effects are observed at $90 \mathrm{~s}$. The temperature of the remaining frost section is marginally below the melting point.

At $t=182.5 \mathrm{~s}$ (Fig. 4d) the frost layer has decreased down to the critical frost layer thickness of $0.2 \mathrm{~mm}$. Now, the remaining frost layer is assumed to drop off, completing the defrost frost process

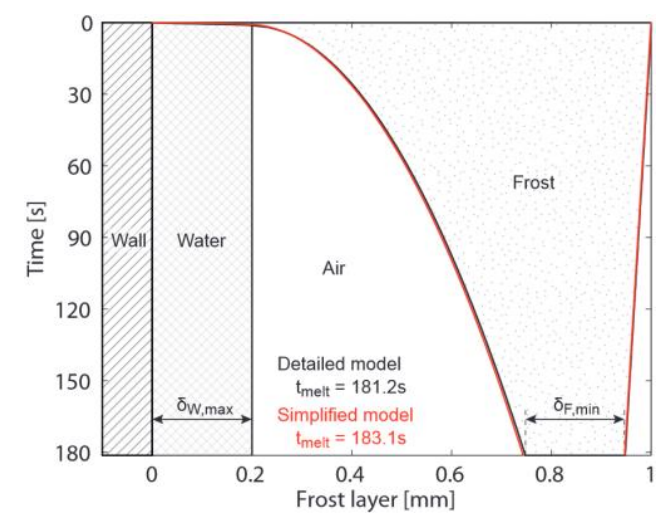

Fig. 5. Remaining frost layer and water and air regions over the defrost time. Black: Presented, detailed model including discretized temperature information of the frost section. Red: Simplified model only considering the latent phase change.

In Fig. 5 the progress of the melting process is plotted over the defrost time (black line) by illustrating the individual regions (melting frost, water film and air gap). The beginning of the defrost process is characterized by wall sided melting in which the water film of critical length develops rapidly. The following increasing air gap acts as an additional thermal baffle, slowing down the process. Thus, the wall sided boundary shows a concave behavior over time. On the other, air-sided frost interface, a linear reduction of the frost layer is observed. The linear defrost behavior at the frost surface indicates an increasing importance of air-sided melting in thicker frost layers, as it is opposed the decreasing wallsided melting process.

In addition to the black lines showing the results of the detailed model (Eq. (1) and (2)), the red line illustrates the results of a further simplified model approach. The approach neglects the local change of temperature, only considering the dominating heat of fusion and the melting process of the frost interface defined by Eq. (2). The simplification is valid due to the high value of the heat of fusion $h_{\text {fus }}=333.5 \mathrm{~kJ} / \mathrm{kg}$ compared to the specific heat capacity of the frost $c_{\mathrm{p}}=1.8 \mathrm{~kJ} /(\mathrm{kg} \mathrm{K})$ and the low increase in temperature in the preheating phase.

Comparing the results of the detailed model (black line) with the simplified approach (red line) a good accordance of the defrost process is observed. Likewise, the calculated defrost times of the frost layer match. In conclusion, the simplified model is likewise able to describe the defrost process, strongly reducing the numerical effort. 


\subsection{Parameter variation}

For further investigation of the defrost model, a parameter variation of the air temperature is performed. The range of $275 \mathrm{~K}$ to $300 \mathrm{~K}$ is chosen for both temperatures, always ensuring a two-sided defrost process. All remaining parameters are adopted from the set-up previously described (compare Tab. 2).

An increase in ambient air temperature accelerates the defrost process. Fig. 6a shows a defrost time of approximately $85 \mathrm{~s}$ at an air temperature of $300 \mathrm{~K}$, whereas, an air temperature slightly above the melting temperature, defrosting takes about $190 \mathrm{~s}$. The convex behavior of the defrost time over the variation of fixed air temperatures as boundary conditions indicates the importance of the air temperature especially close to the melting point. In Fig. 6b, the location of the remaining frost layer before its thickness undercuts the critical stability criteria is displayed. With an increasing air temperature, the remaining frost layer moves from the initial air interface towards the wall.

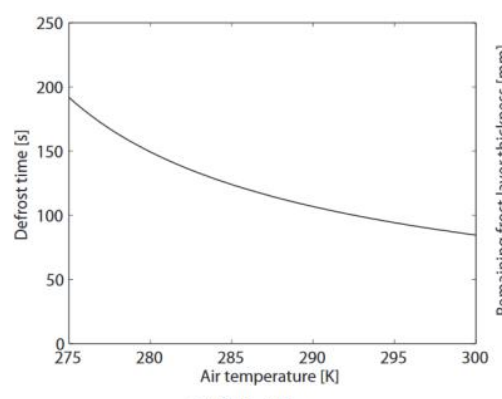

(a) Defrost time

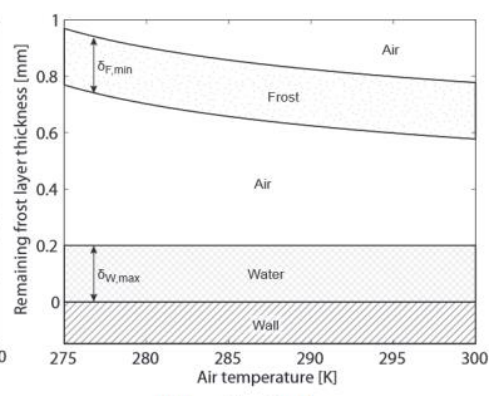

(b) Remaining frost layer

Fig. 6. Variation of the air temperature $T_{a}$.

\section{Conclusion}

A numerical approach for a one-dimensional transient defrost model accounting for heat conduction is presented. Fixed boundary conditions are applied to the frost interfaces. This simplified approach allows for an integration of the defrost model in a CFD solver (OpenFOAM) using a zero-dimensional wall function. In accordance to previous work, a multi stage model is applied (e.g. [1,2]).

For validation and verification, the multi-stage model is implemented in MATLAB, evaluating the defrost process of a one-dimensional frost segment. The simulation results verify the plausibility of the designed model. The evaluation of the simulated defrost process shows the expected behavior of the three-stage sequence. Likewise, the variation of boundary conditions results in plausible reactions from the defrost model. A convergent behavior of the numerical implementation is also conducted.

W.R. is associated postdoctoral researcher in the graduate school GRK 1856 "mobilEM" funded by the German Research Foundation (DFG).

\section{References}

1. C. Sanders, Ph.D. thesis, University of Delft (1974)

2. J.A. Dopazo, J. Fernandez-Seara, F.J. Uhia, R. Diz, Int. J. of Refrigeration 33, 829-839 (2010)

3. Y. Hayashi, A. Aoki, S. Adachi, K. Hori, J. Heat Transfer 99, 239-245 (1977) 
4. S. Anisimov, A. Jedlikowski, D. Pandelidis, Int. J. Heat Mass Transfer 90, 201-217 (2015)

5. X. Wu, S. Hu, F. Chu, Applied Thermal Engineering 95, 95-105 (2016)

6. A. Alebrahim, S. Sherif, J. of Mechanical Engineering Science 216, 655-673 (2002)

7. N. Hoffenbecker, Master's thesis, University of Wisconsin (2004)

8. N. F. Aljuwayhel, Ph.D. thesis, University of Wisconsin (2006)

9. W. F. Mohs, Ph.D. thesis, University of Minnesota (2013)

10. C. Machielsen, H. Kerschbaumer, Int. J. of Refrigeration 12, 283-290 (1989)

11. N. Hoffenbecker, S. Klein, D. Reindl, Int. J. of Refrigeration 28, 605-615 (2005)

12. B. Wellig, M. Imholz, M. Albert, K. Hilfiker, 9th IEA Heat Pump Conference, Zurich $\mathrm{CH}(2008)$

13. S. Sherif, M. Hertz, Int. J. of Energy Research 22, 85-92 (1998)

14. S. Bertsch, P. Hubacher, M. Ehrbar, SFOE (2002)

15. B. Hubacher, M. Ehrbar, SFOE (2000)

16. P. Hubacher, C. Bernal, M. Ehrbar, S. Schwendener, SFOE (2004)

17. X. Wu, S. Hu, F. Chu, Applied Thermal Engineering 95, 95-105 (2016)

18. F. Wang, C. Liang, M. Yang, C. Fan, X. Zhang, Applied Thermal Engineering 75, 1126-1132 (2015)

19. M. Kandula, Tech. rep., ASRCAerospace, John F. Kennedy Space Center, FL, USA (2010)

20. K. Prölss, G. Schmitz, The Modelica Association 1, 509-516 (2006) 